\title{
MondoA coordinately regulates skeletal myocyte lipid homeostasis and insulin signaling
}

\author{
Byungyong Ahn, ${ }^{1}$ Mangala M. Soundarapandian, ${ }^{1}$ Hampton Sessions, ${ }^{2}$ Satyamaheshwar Peddibhotla, ${ }^{2}$ Gregory P. Roth, ${ }^{1}$ \\ Jian-Liang Li, ${ }^{1}$ Eliot Sugarman, ${ }^{2}$ Ada Koo, ${ }^{2}$ Siobhan Malany, ${ }^{2}$ Miao Wang, ${ }^{1}$ Kyungmoo Yea, ${ }^{1}$ Jeanne Brooks, ${ }^{1}$ Teresa C. Leone, ${ }^{1}$ \\ Xianlin Han, ${ }^{1}$ Rick B. Vega, ${ }^{1}$ and Daniel P. Kelly'
}

${ }^{1}$ Center for Metabolic Origins of Disease and ${ }^{2}$ Conrad Prebys Center for Chemical Genomics, Sanford Burnham Prebys Medical Discovery Institute at Lake Nona (SBP-LN), Orlando, Florida, USA.

\begin{abstract}
Intramuscular lipid accumulation is a common manifestation of chronic caloric excess and obesity that is strongly associated with insulin resistance. The mechanistic links between lipid accumulation in myocytes and insulin resistance are not completely understood. In this work, we used a high-throughput chemical biology screen to identify a small-molecule probe, SBI-477, that coordinately inhibited triacylglyceride (TAC) synthesis and enhanced basal glucose uptake in human skeletal myocytes. We then determined that SBI-477 stimulated insulin signaling by deactivating the transcription factor MondoA, leading to reduced expression of the insulin pathway suppressors thioredoxin-interacting protein (TXNIP) and arrestin domain-containing 4 (ARRDC4). Depleting MondoA in myocytes reproduced the effects of SBI-477 on glucose uptake and myocyte lipid accumulation. Furthermore, an analog of SBI-477 suppressed TXNIP expression, reduced muscle and liver TAC levels, enhanced insulin signaling, and improved glucose tolerance in mice fed a high-fat diet. These results identify a key role for MondoA-directed programs in the coordinated control of myocyte lipid balance and insulin signaling and suggest that this pathway may have potential as a therapeutic target for insulin resistance and lipotoxicity.
\end{abstract}

\section{Introduction}

The rising prevalence of obesity is driving an alarming increase in type 2 diabetes, a global health threat. Comorbidities associated with obesity include insulin resistance and fatty liver disease (nonalcoholic fatty liver disease and nonalcoholic steatohepatitis [NAFLD/NASH]). The development of obesity-related diabetes represents a final common pathogenic pathway that further contributes to the comorbid diseases. Accordingly, delineation of the mechanisms involved in the development of insulin resistance is a critical step toward the identification of new therapeutic targets aimed at the early treatment of this progressive, feed-forward disease process.

The development of insulin resistance is strongly associated with accumulation of intracellular lipid in tissues outside of adipose including skeletal muscle, liver, and heart. In obese humans, intramyocellular lipid (IMCL) is negatively correlated with wholebody insulin sensitivity (1-3). The skeletal myocyte imports FAs into the cell from circulating free fatty acids (FFAs) or lipoprotein particles, such as very low-density lipoprotein (VLDL), to support

\footnotetext{
Authorship note: G.P. Roth is deceased.
}

Note regarding evaluation of this manuscript: Manuscripts authored by scientists associated with Duke University, The University of North Carolina at Chapel Hill, Duke-NUS, and the Sanford-Burnham Prebys Medical Discovery Institute are handled not by members of the editorial board but rather by the science editors, who consult with selected external editors and reviewers.

Conflict of interest: D.P. Kelly is a scientific consultant for Pfizer Inc. and receives research support from Takeda Pharmaceutical Co. R.B. Vega receives research support from Pfizer Inc.

Submitted: March 8, 2016; Accepted: June 9, 2016

Reference information: J Clin Invest. 2016;126(9):3567-3579. doi:10.1172/JCI87382. energy production. Once transported into the cell, FAs are oxidized for ATP production, used to build membranes, or stored as triacylglycerides (TAGs). However, excessive skeletal myocyte lipid delivery, such as occurs in the obese state, leads to expansion of IMCL. Increased import of FAs is thought to initially trigger an adaptive response within the skeletal muscle to increase the capacity for mitochondrial FA oxidation (FAO) (4). In the long term, however, increased delivery of FAs can exceed mitochondrial oxidative capacity, setting the stage for a "vicious cycle" of cellular lipotoxicity and leading to insulin resistance. In support of this notion, some studies have shown that mitochondrial oxidative capacity is reduced in insulin-resistant diabetic subjects (5-7).

The mechanistic links between IMCL and the development of insulin resistance is poorly understood. The results of studies to date suggest that the lipid storage depot, per se, is probably not a culprit in the genesis of cellular "lipotoxicity" and insulin resistance. Indeed, some studies have suggested that the capacity to store lipids within the cell serves a protective function $(8,9)$. Rather, the generation and accumulation of lipid intermediates have been proposed to alter insulin-stimulated glucose uptake $(10,11)$. For example, lipid-derived diacylglycerol (DAG) species have been shown to activate protein kinase $C-\varepsilon$ and $\theta$ isoforms to phosphorylate the insulin receptor substrate 1 (IRS-1), blocking the actions of the insulin receptor $(12,13)$. Ceramides and ROS have also been shown to inhibit insulin signaling in certain contexts $(14,15)$. In addition, intermediates of incomplete FAO have been implicated in insulin resistance (16). However, the role of such processes as primary drivers of insulin resistance related to altered cellular lipid balance versus that of serving as downstream effectors is unclear. Moreover, regulatory circuitry that 
A

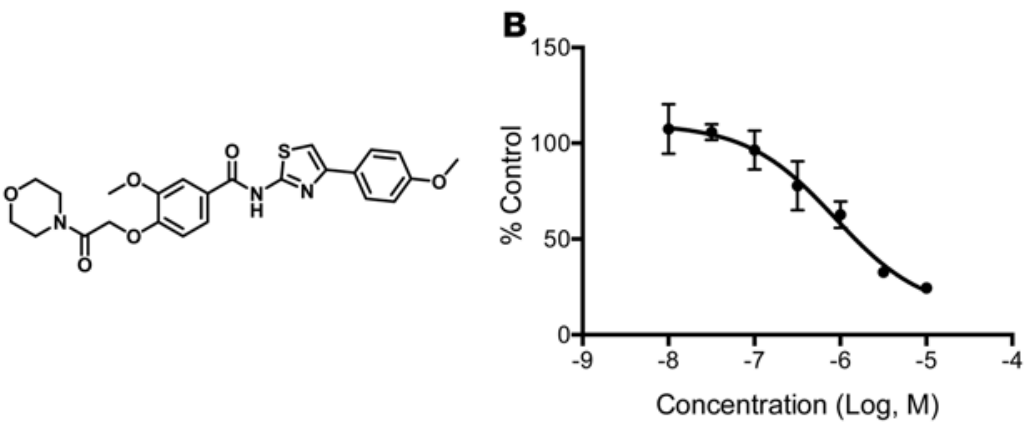

C

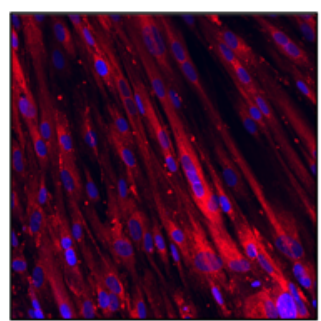

BSA/vehicle

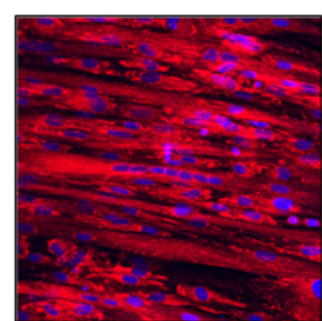

Oleate/vehicle

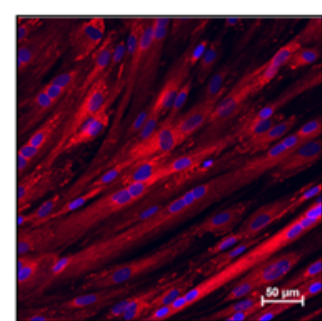

Oleate/SBI-477
Figure 1. SBI-477 is a small-molecule inhibitor of neutral lipid accumulation in human skeletal myotubes. (A) Chemical structure of SBI-477. (B) Effects of SBI-477, over a dose range, on triglyceride levels in human skeletal myotubes following a 24-hour exposure to $100 \mu \mathrm{M}$ oleate. Data are shown as the percentage of DMSO vehicle control and are representative of more than 5 experiments. (C) Effects of SBI-477 on human skeletal myotube neutral lipid accumulation as visualized by AdipoRed staining (red). Cell nuclei were stained with DAPI (blue). Images shown are representative of more than 3 images. links the control of cellular lipid balance and insulin signaling has not been identified. Delineation of such upstream mechanisms could lead to the identification of new therapeutic targets for the treatment of insulin resistance.

We have recently pursued an unbiased strategy, in which a high-throughput chemical biology screen was used to identify small-molecule probes that influence downstream pathways involved in the control of cellular neutral lipid stores (17). As described herein, one such molecule, SBI-477, coordinately reduced myocyte lipid stores and increased glucose uptake. We show that SBI-477 is a potent inhibitor of FA incorporation into TAGs in human skeletal myocytes. In parallel, SBI-477 increases myocyte glucose uptake by activating insulin signaling. The cellular actions of SBI-477 are attributable, at least in part, to inhibition of the transcription factor MondoA, resulting in reduced expression of TAG synthesis genes and suppressed transcription of genes encoding suppressors of insulin signaling.

\section{Results}

Identification of a small-molecule inhibitor of myocyte neutral lipid accumulation. A cell-based high-throughput screen was performed previously in murine AML12 hepatocytes to identify molecular probes that decrease TAG accumulation resultant from oleate loading (17). Hits from this screen were examined for chemical tractability and activity in human skeletal myocytes. One particular compound, an $\mathrm{N}$-(thiazol-2-yl)-benzamide termed SBI-477 (Figure 1A), showed potent inhibition of TAG accumulation in rat $\mathrm{H} 9 \mathrm{c} 2$ myocytes (half-maximal effective concentration $\left[\mathrm{EC}_{50}\right] \approx 100 \mathrm{nM}$, data not shown) and human skeletal myotubes $\left(\mathrm{EC}_{50} \approx 1 \mu \mathrm{M}\right.$; Figure 1, B and C, and Supplemental Figure 1A; supplemental material available online with this article; doi:10.1172/ JCI87382DS1). Inhibition of TAG accumulation by SBI-477 was not due to blocking cellular FA uptake or increasing intracellular lipolysis rates (Supplemental Figure 1B and data not shown). In addition, SBI- 477 had no effect on the gene expression of the
FA transporter CD36 (data not shown). Increased oxidation of FAs could also account for the TAG-lowering actions of SBI-477. Indeed, FAO rates were increased by SBI- 477 in a dose-dependent manner that was concordant with inhibition of increased TAG storage (Supplemental Figure 2A). Acylcarnitine species indicative of mitochondrial FAO intermediates were also increased following exposure to SBI-477 in human myotubes, consistent with increased mitochondrial FAO rates (Supplemental Figure 2B). However, inhibition of FAO did not prevent the actions of SBI-477 on TAG levels, even in the presence of carnitine, suggesting that this effect is likely downstream of the primary site of TAG-lowering action (Supplemental Figure 2, B and C).

To assess the effects of SBI-477 on triglyceride synthesis and remodeling, quantitative lipidomic analyses were conducted on extracts of oleate-loaded human skeletal myotubes following a 24-hour exposure to SBI-477 and compared with vehicle (Supplemental Tables 1 and 2). The effects of SBI-477 were broad, reducing all TAG species measured, with the greatest effects on TAG species with acyl chain lengths from 16 to 20 . We observed a marked reduction of $18: 1$ species by SBI-477, consistent with oleate loading (Figure 2, A and B). Levels of TAG and DAG species were also reduced following exposure to the compound (Figure 2A and Supplemental Tables 1 and 2). We also examined the levels of specific lipid species implicated in insulin resistance or cellular lipotoxicity. Levels of ceramide and total sphingomyelin were not significantly altered with SBI-477 treatment in oleate-loaded myotubes (Supplemental Table 1). However, a significant reduction in a subset of sphingomyelin species, including those containing a 16:0 acyl chain, was observed with SBI- 477 treatment. Interestingly, however, these changes were not observed in nonoleate-loaded myotubes (Supplemental Table 2). These results indicate that SBI477 inhibits the incorporation of all FAs, including those of exogenous origin, into the neutral lipid TAG pool.

Intracellular TAG can be formed through reacylation of DAG species generated from several pathways including a de novo 

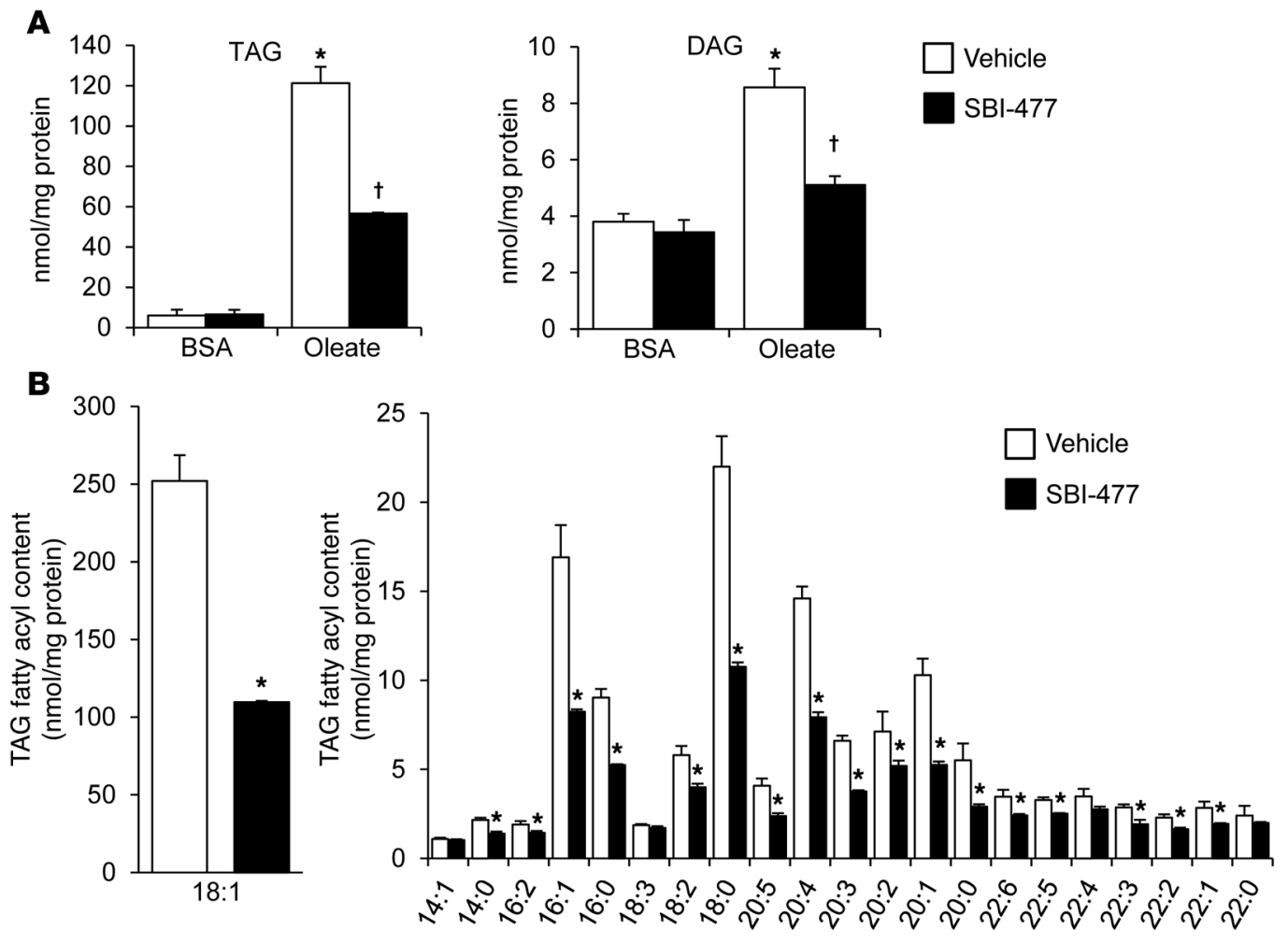

Figure 2. SBI-477 inhibits expansion of cellular DAG and TAG pools in oleate-loaded myocytes. Results of quantitative lipidomic analyses performed on human skeletal myotubes exposed to BSA or $100 \mu \mathrm{M}$ oleate in the presence of DMSO vehicle (white bars) or $10 \mu \mathrm{M}$ SBI-477 (black bars) for 24 hours. (A) Total mean TAG and DAG levels are shown. (B) Levels of individual TAG fatty acyl species, with 18:1 species shown separately (left). Data represent the mean $\pm \operatorname{SD}(n=3) .{ }^{*} P<0.05$ versus vehicle; ${ }^{\dagger} P<0.05$ versus oleate-loaded vehicle; Student's $t$ test.

glycerolphosphate biosynthetic pathway that involves dephosphorylation of phosphatidic acid, reacylation of monoacylglycerol, and, to a lesser extent, hydrolysis of phosphatidyl inositol. To explore the effects of SBI-477 on specific TAG biosynthesis pathways, a bioinformatics lipidomics modeling approach was used as previously described (18). This approach uses the individual TAG ion profile, as determined by mass spectrometry, to predict the relative contribution of each pathway to the overall cellular TAG pool. Experiments were conducted in skeletal myocytes in the absence and presence of oleate loading. In the absence of exogenous oleic acid, SBI-477 markedly reduced the contribution by the de novo glycerolphosphate biosynthetic pathway (K1), resulting in a shift (10-fold increase) to the monoacylglycerol reacylation pathway (K2) (Supplemental Table 3). In contrast, incubation with SBI-477 resulted in a modest decrease in $\mathrm{K} 2$, with a shift to $\mathrm{K} 1$ in the oleate-loaded condition. These results, together with the lipidomic profiling data, indicate that SBI-477 inhibits several cellular TAG synthesis pathways and that the mechanism for inhibition of TAG biosynthesis by SBI477 is distinct, depending on whether the FA source is exogenous or synthesized de novo. Notably, we did not find that SBI-477 directly inhibits the activity of stearoyl-coenzyme A desaturase 1 (SCD1), diacylglycerol $O$-acyltransferase $1 / 2$ (DGAT1/2), or monoacylglycerol $O$-acyltransferase 1/2/3 (MGAT1/2/3) (data not shown), suggesting a novel inhibitory mechanism.

Stimulation of muscle glucose uptake and insulin signaling by SBI-477. We next sought to determine whether the IMCL-lowering effects of the molecular probe SBI-477 was linked to changes in myocyte glucose uptake. SBI-477 increased both basal and insulin-stimulated glucose uptake in human skeletal myotubes (approximately $84 \%$ at $10 \mu \mathrm{M}$ SBI-477; Figure 3A). Glycogen synthesis rates were also enhanced by SBI-477 treatment (Figure 3B). The effects on glucose uptake were independent of, but additive with, that of insulin. Similar effects on glucose uptake were observed in oleate-loaded myotubes (Supplemental Figure 3A). Interestingly, SBI-477 activated insulin signaling in the absence of insulin. Specifically, tyrosine phosphorylation of the insulin receptor substrate-1 (IRS-1) was increased by SBI-477 (Figure 3, C and D). Conversely, IRS-1 phosphorylation at serine sites 636 and 639, which has been shown to confer inhibition of insulin signaling (19), was decreased by exposure to SBI-477. Phosphorylation of the downstream effector kinase Akt was also increased by SBI- 477 . The effects on insulin signaling triggered by SBI- 477 were observed after 24 hours of compound exposure, but not with acute treatment (data not shown). These results indicate that SBI- 477 stimulates glucose uptake by activating insulin signaling through a mechanism that does not require insulin engagement to its receptor.

We also assessed signaling events downstream of insulin signaling. Surprisingly, levels of phosphorylated S6K (p-S6K) (T389), a target of insulin/mTORC1 signaling, were reduced by SBI-477 treatment (Supplemental Figure 3B). We, therefore, examined other signaling pathways upstream of mTORC1. Notably, SBI-477 treatment resulted in activation of AMPK, an inhibitor of mTOR signaling, consistent with the observed inhibitory phosphorylation of S6K (Supplemental Figure 3B). It is possible, therefore, that 


\section{A}

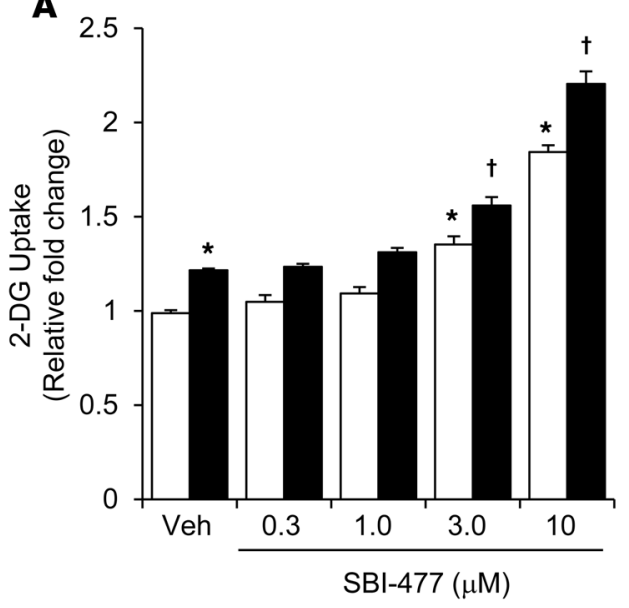

C

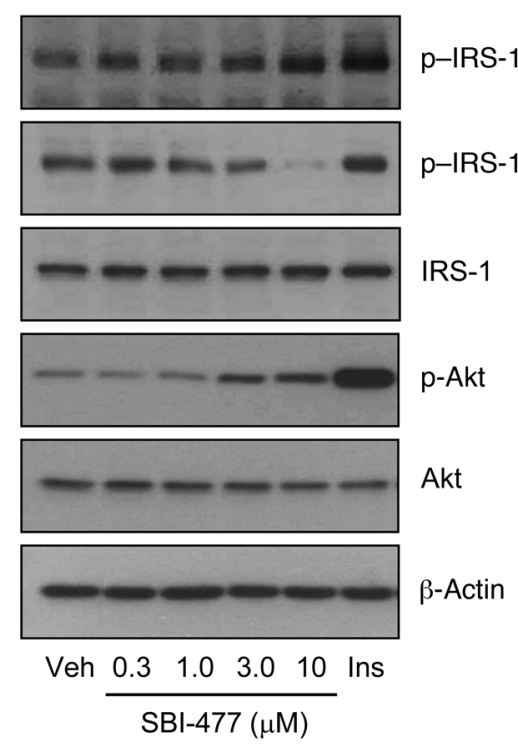

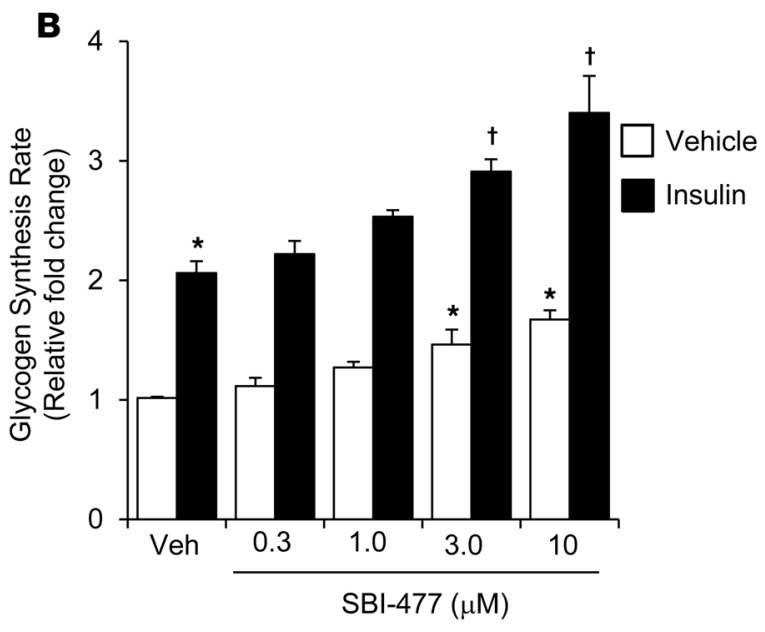
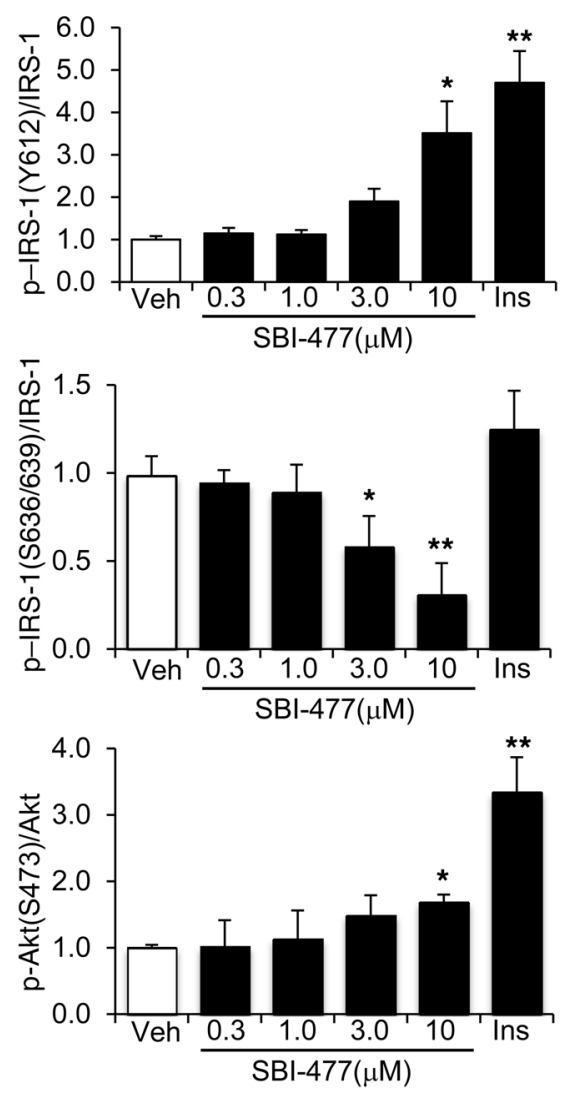

Figure 3. SBI-477 stimulates glucose uptake and activates insulin signaling in the absence of insulin. Human skeletal myotubes were incubated with SBI-477 at the indicated concentration for 24 hours and then treated with or without insulin ( $100 \mathrm{nM})$ for 30 minutes. Glucose (2-DG) uptake (A) and glycogen synthesis rates (B) were measured $(n=5)$ as described in Methods. (A and $\mathbf{B}){ }^{*} P<0.05$ versus vehicle, no insulin; ${ }^{\dagger} P<0.05$ versus vehicle with insulin; 2-way ANOVA with Tukey's post-hoc test. (C) Western blot analysis of human myotubes treated with SBI-477 for 24 hours was performed to determine the effect on steps of the insulin-signaling pathway using specific Akt and IRS-1 phosphorylation sites as endpoints. Insulin treatment (100 nM) for 30 minutes was used as a positive control. (D) Graph shows quantitation of the Western blot data in $\mathbf{C}(n=5)$. ${ }^{*} P<0.05$ and ${ }^{* *} P<0.01$ versus vehicle; 1-way ANOVA with Bonferroni's post-hoc test. Data represent the mean \pm SD. Ins, insulin; Veh, vehicle.

activation of AMPK inhibits mTORC1 activity, despite activation of insulin signaling, in the context of SBI-477 exposure.

SBI-477 reduces expression of TXNIP and ARRDC4, negative regulators of insulin signaling, via deactivation of the transcription factor MondoA. To gain further insight into the downstream actions of SBI477 , we conducted transcriptional profiling in primary human skel- etal myotubes. Heatmap visualization of differentially expressed transcripts revealed that a 24-hour exposure to SBI-477 prevented many of the transcriptomic changes resultant from oleate loading and regulated a distinct subset of transcripts compared with that of a DGAT1 inhibitor (Supplemental Figure 4). Examination of the transcripts regulated by SBI-477, but not the DGAT inhibitor, 
A

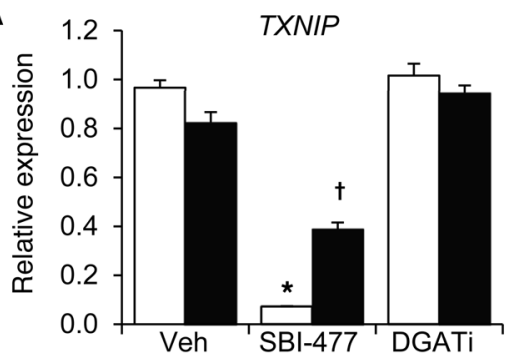

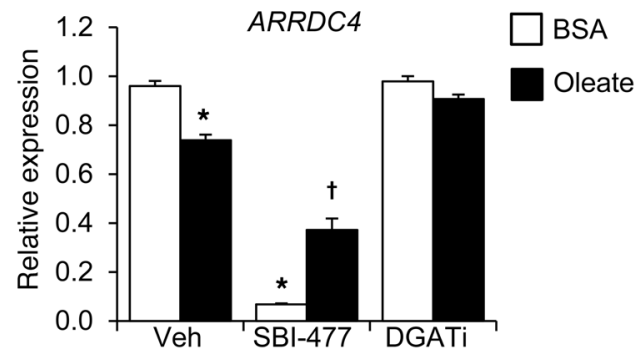

B

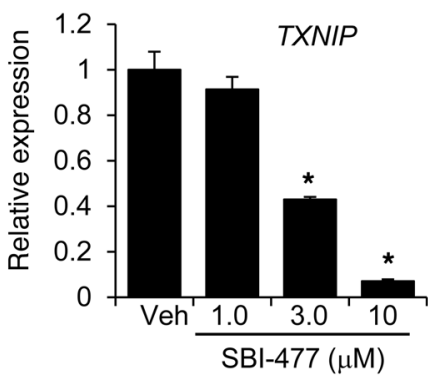

C
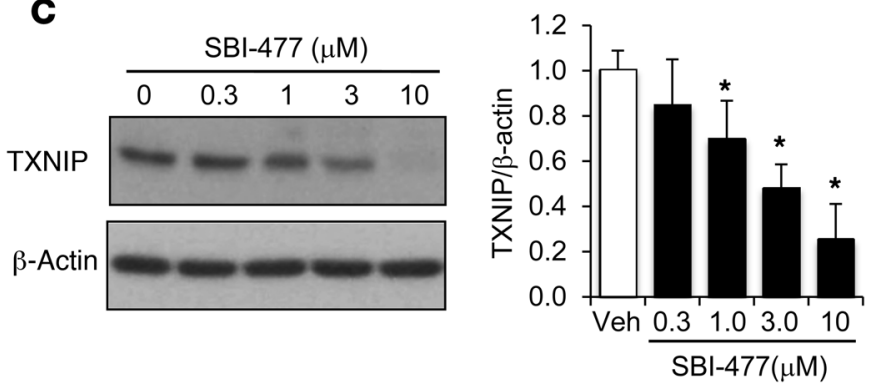

Figure 4. Downregulation of TXNIP and ARRDC4 expression by SBI-477. (A) TXNIP and ARRDC4 mRNA levels as determined by quantitative reverse transcription PCR (qRT-PCR) in human myotubes treated with SBI-477 (10 $\mu \mathrm{M}$ ) or DCAT1 inhibitor (DCATi, $1 \mu \mathrm{M})$ for 24 hours in the absence or presence of $100 \mu \mathrm{M}$ oleate $(n=4)$. Expression is shown relative to vehicle with BSA treatment. (B) TXNIP gene expression following exposure to a dose range of SBI-477 for 24 hours $(n=4)$. (C) Western blot analysis was performed to determine the effect of SBI-477 on TXNIP protein levels. Graph shows quantitation of the TXNIP Western blot data $(n=5)$. ${ }^{*} P<0.05$ versus vehicle/BSA control; ${ }^{\dagger} P<0.05$ versus vehicle/oleate; 1 -way ANOVA with Bonferroni's post-hoc test. Data represent the mean \pm SD.

identified thioredoxin-interacting protein (TXNIP) and arrestin domain-containing 4 (ARRDC4) as markedly downregulated. TXNIP and ARRDC4 have been shown to function as potent negative regulators of glucose uptake and insulin signaling $(20,21)$. SBI477 conferred robust, dose-dependent downregulation of TXNIP and ARRDC4 expression in human myotubes, an effect that was also observed under oleate-loaded conditions, albeit to a lesser extent (Figure 4, A and B). TXNIP protein levels were also reduced by SBI-477 in a dose-dependent manner (Figure $4 \mathrm{C}$ ).

Given the marked downregulation of TXNIP and ARRDC4 transcript levels by SBI-477, we next explored effects at the transcriptional level. For these studies, a reporter construct containing approximately $1.5 \mathrm{~kb}$ of the human TXNIP promoter was transfected into H9c2 myocytes (22). SBI-477 decreased promoter activity in a dose-dependent manner (Figure 5A). TXNIP expression is known to be regulated, at least in part, by 2 carbohydrate response elements (ChoREs) within its promoter region (23). Mutational analysis confirmed that SBI-477-mediated inhibition of the TXNIP promoter is dependent on an intact ChoRE (Figure 5B).

ChoREs were originally characterized as being bound by heterodimers composed of the carbohydrate-responsive element-binding protein (ChREBP, also termed MondoB) and Maxlike protein $\mathrm{X}(\mathrm{MLX})$ transcription factors. A highly related protein, MondoA, is muscle enriched and has been shown to bind to similar consensus sites (24). We confirmed by immunoblotting that human myotubes in culture predominantly express MondoA compared with ChREBP expression (data not shown). Both ChREBP and MondoA have been shown to regulate TXNIP and ARRDC4 expression $(23,25)$. Therefore, we sought to determine whether SBI- 477 affected the occupation of the ChoREs located in the TXNIP and ARRDC4 promoter regions. ChIP studies confirmed the occupation of MondoA on ChoREs located within both the TXNIP and ARRDC4 promoter regions, but not on a control myocyte enhancer factor 2-binding (MEF2-binding) site in the inositol(myo)-1(or 4)-monophosphate 2 (IMPA2) gene promoter in human skeletal myotubes (Figure 5C). The occupation by MondoA was inhibited by SBI-477 (Figure $5 \mathrm{C}$ ). Thus, SBI-477 reduces the binding of MondoA to the TXNIP and ARRDC4 promoter regions.

The activity of ChREBP and MondoA is governed, in part, by nuclear-cytoplasmic shuttling mechanisms that are incompletely understood but probably involve glucose metabolites and phosphorylation $(25,26)$. Accordingly, we assessed the effects of SBI-477 on intracellular localization of MondoA. Immunolocalization studies confirmed that treatment with SBI-477 resulted in near-complete nuclear exclusion of MondoA in human skeletal myoblasts (Figure 5D). The results of the immunoblotting studies conducted with fractionated cellular extracts further supported this conclusion (Figure 5E). Taken together, these results demonstrate that downregulation of TXNIP and ARRDC4 expression downstream of SBI-477 involves deactivation of MondoA via effects on nuclear localization.

MondoA coordinately regulates pathways involved in myocyte glucose uptake and TAG synthesis. siRNA-mediated knockdown (KD) studies were performed in human skeletal myocytes to determine whether inhibition of MondoA was responsible for the observed effects of SBI-477 on myocyte glucose uptake. Following MondoA (MLXIP) KD (Supplemental Figure 5), expression of TXNIP and ARRDC4 was reduced to a degree similar to that detected with SBI-477 treatment (Figure 6, A and B). Insulin-independent glucose uptake was enhanced following MondoA KD in human myotubes (Figure 6C). 


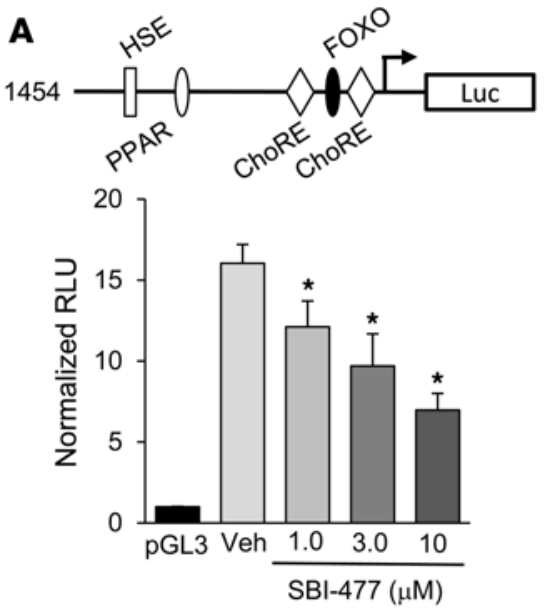

B

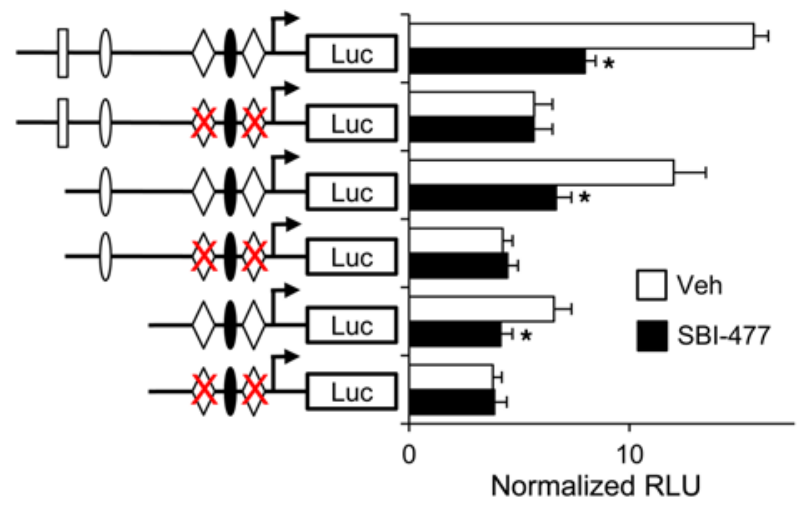

C TXNIP ChoRE

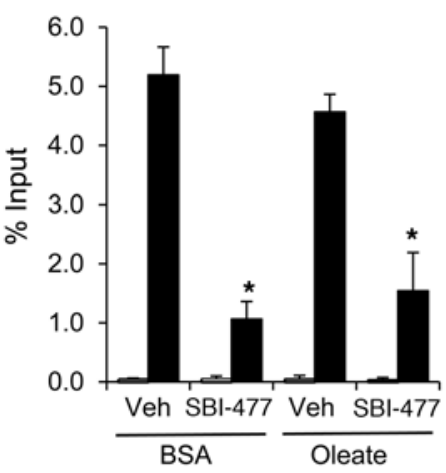

ARRDC4 ChoRE

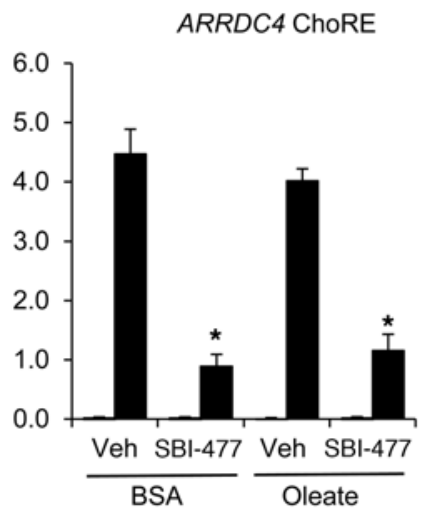

IMPA2

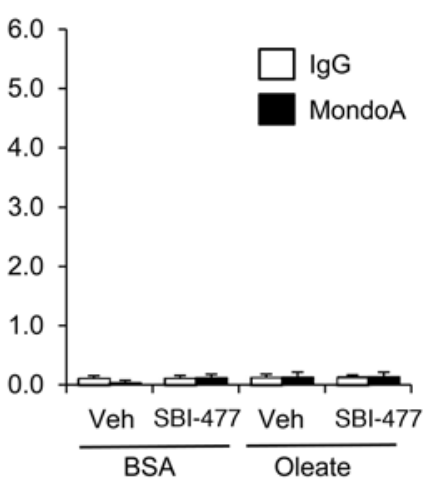

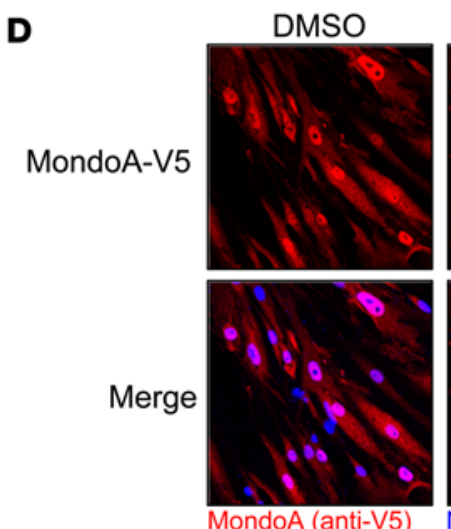

MondoA (anti-V5)

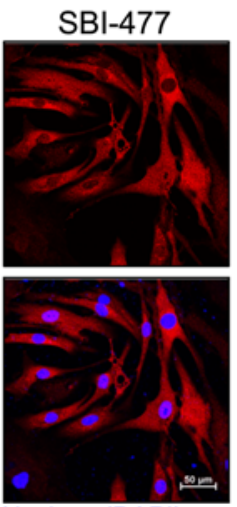

Nucleus (DAPI)
$\mathbf{E}$

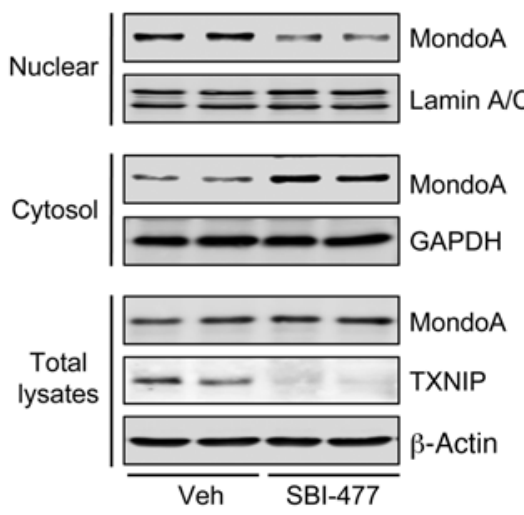

Figure 5. SBI-477 inhibits MondoA-mediated activation of the TXNIP gene promoter via effects on nuclear localization. (A) A luciferase reporter construct containing approximately $1.5 \mathrm{~kb}$ of the human TXNIP promoter (schematic) or a pGL3 control vector was transfected into H9c2 skeletal myocytes. The activity of the TXNIP promoter (relative luciferase units [RLU]) was measured following treatment with SBI-477 at the indicated concentration $(n=5)$. (B) The activity of WT versus ChoRE mutant TXNIP promoters was measured in the presence and absence of SBI-477 (10 $\mu$ M) for 24 hours $(n=5)$. The red "X" denotes inactivity mutations. (C) ChIP-qRT-PCR analysis was performed with anti-MondoA (black bars) or IgG (white bars) control Abs in human skeletal myotubes. Occupancy of MondoA on the ChoRE of the TXNIP or ARRDC4 promoters following treatment with SBI-477 in the absence or presence of oleate is shown $(n=4)$. Occupation on a MEF2-binding site within the IMPA2 promoter was used as a negative control. (D) Representative images of more than 3 micrographs of MondoA-V5 in human skeletal myoblasts following treatment with $10 \mu \mathrm{M}$ SBI-477 or DMSO vehicle control for 24 hours. MondoA-V5 staining is shown in red, DAPI in blue. (E) Western blot analysis was performed on total cell lysates and nuclear or cytoplasmic fractions from primary human myotubes treated with $10 \mu \mathrm{M} \mathrm{SBI-477} \mathrm{or} \mathrm{DMSO} \mathrm{vehicle} \mathrm{control} \mathrm{for} 24$ hours. Lamin A/C and CAPDH were included as controls for the nuclear and cytoplasmic fractions, respectively. ${ }^{*} P<0.05$ versus vehicle; 1-way ANOVA with Bonferroni's post-hoc test. Data represent the mean \pm SD. HSE, heat shock element; Luc, luciferase. 
A
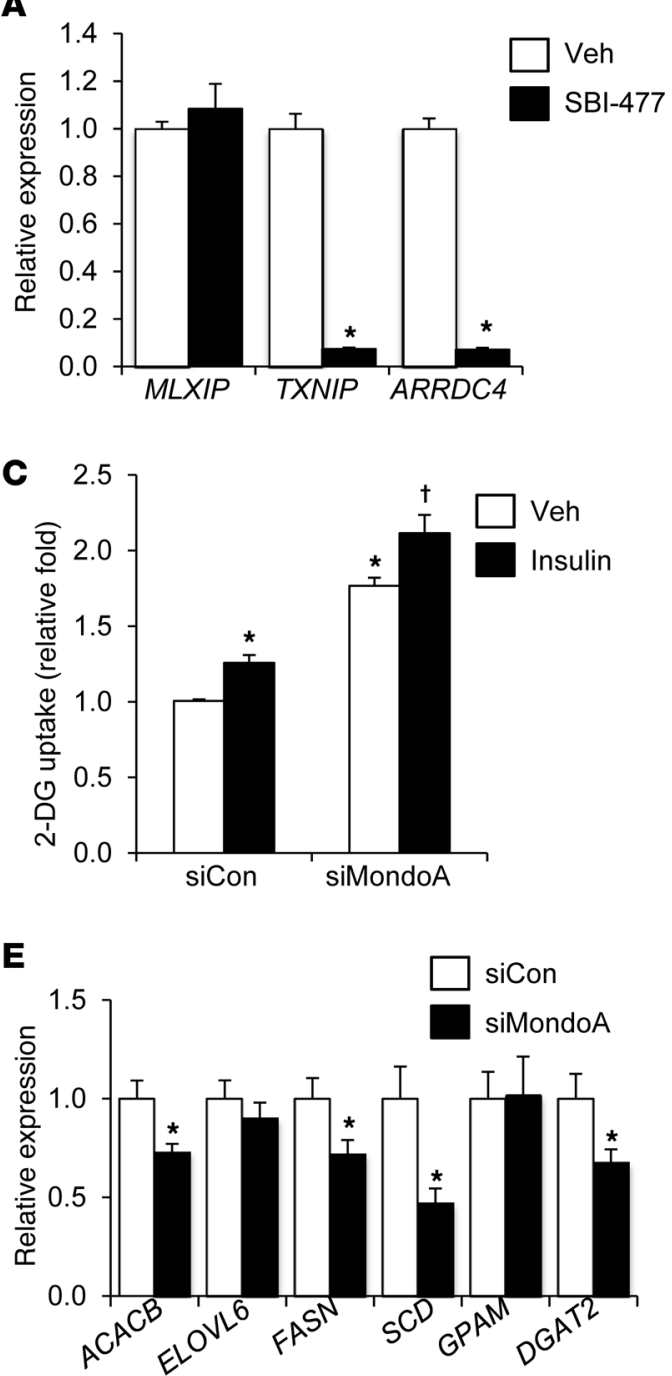

B

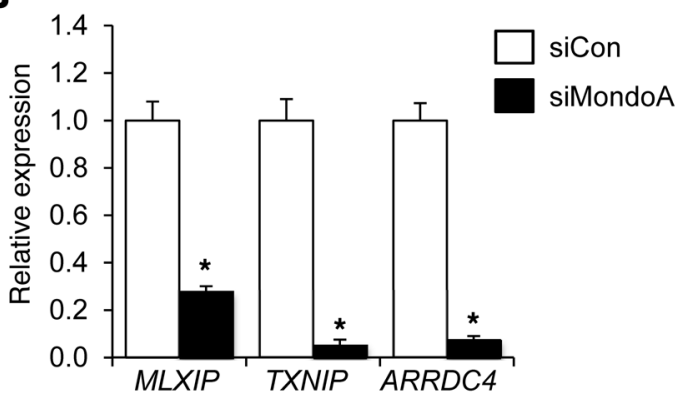

D

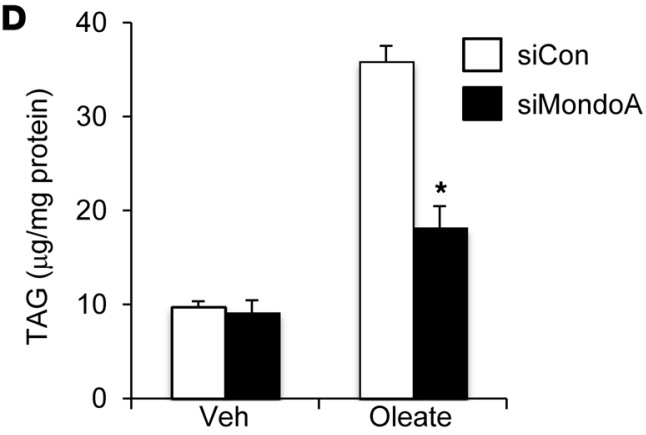

$\mathbf{F}$

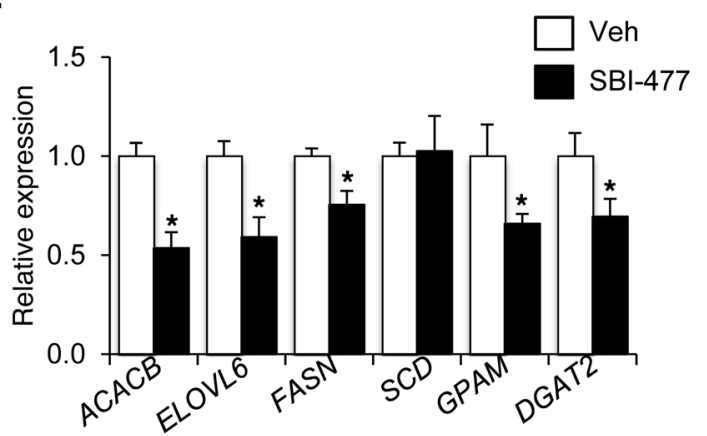

Figure 6. MondoA depletion mimics action of SBI-477 in human skeletal myotubes. TXNIP and ARRDC4 gene expression was measured following treatment with $10 \mu \mathrm{M} \mathrm{SBI}-477$ (A) or siRNA-mediated MondoA $(M L X I P) \operatorname{KD}(B)(n=4) .{ }^{*} P$ $<0.05$ versus vehicle $(A)$ or nontargeting siRNA control (siCon) (B). (C) 2-DG uptake following MondoA KD (or siCon) in the absence or presence of insulin $(n=5)$. ${ }^{*} P<0.05$ versus siCon/vehicle; ${ }^{\dagger} P<0.05$ versus siCon/ insulin. (D) Cellular TAC levels following MondoA KD in the absence or presence of $100 \mu$ M oleate $(n=5)$ ${ }^{*} P<0.05$ versus siCon/ oleate. Effect of MondoA $\mathrm{KD}(\mathrm{E})$ or $\mathrm{SBI}-477$ treatment (F) on the expression of genes encoding lipogenic and triglyceride synthesis enzymes $(n=4) .{ }^{*} P<0.05$ versus vehicle or siCon. Data represent the mean \pm SD. Statistical significance in all panels was determined by Mann-Whitney $U$ test.
MondoA KD also inhibited TAG accumulation following oleate loading (Figure 6D). Given that our lipidomic studies implicated a mechanism targeting TAG synthesis, we sought to determine whether the SBI-477/MondoA pathway affected the expression of genes involved in this pathway. MondoA KD reduced the expression of several genes, including FASN, GPAM, ACACB (also known as ACC2), and DGAT2, in the lipogenesis and TAG synthesis pathways (Figure 6E). A very similar, although not identical, gene-regulatory pattern was observed with SBI-477 treatment (Figure 6F). Thus, depletion of MondoA reproduced the actions of SBI-477 on both glucose and lipid metabolism.

To determine whether the effects conferred by SBI- 477 and observed in myocytes in culture are relevant in vivo, we conducted studies in C57BL/6 mice with diet-induced obesity. For these studies, we used an analog of SBI-477, named SBI-993, that showed improved potency and suitable pharmacokinetic properties for in vivo bioavailability (Supplemental Figure 6, A and B). SBI-993 reduced Txnip and Arrdc4 expression to a degree similar to that seen with SBI-477 in human myotubes (data not shown). Mice were fed a $60 \%$ high-fat diet (HFD) for 8 weeks, which resulted in significant weight gain (Supplemental Figure 6C). During the final week of HFD feeding, SBI-993 or vehicle control was admin- istered as a once-daily dose $(50 \mathrm{mg} / \mathrm{kg}$ s.c.) for 7 days. Plasma concentrations of SBI-993 $(4.97 \pm 0.97 \mu \mathrm{M}) 4$ hours following the final dose were above the cellular $\mathrm{EC}_{50}$. SBI-993 treatment resulted in a small but significant reduction in BW as compared with vehicle treatment (Supplemental Figure 6C).

The effect of SBI-993 on MondoA target gene expression was used as a biomarker to confirm its expected actions in vivo. SBI-993 treatment reduced the expression of TAG synthesis and lipogenic genes in both muscle and liver (Figure 7A). In addition, SBI-993 administration reduced Txnip and Arrdc4 expression, an effect that was especially robust in liver (Figure 7A). ChIP analysis demonstrated that occupation of both ChREBP and MondoA on the Txnip and pyruvate kinase (Pklr) gene promoters was reduced in liver by SBI-993 (Supplemental Figure 7).

Consistent with the observed actions in vitro, TAG levels were significantly reduced in skeletal muscle following SBI-993 administration (Figure 7B). Hepatic steatosis was also substantially ameliorated with compound treatment (Figure 7B and Supplemental Figure 8A). The decreased lipid deposition in liver and skeletal muscle was associated with improved glucose tolerance in mice administered SBI-993 (Figure 7C). Finally, SBI-993 improved insulin signaling in both muscle and liver following an acute insu- 

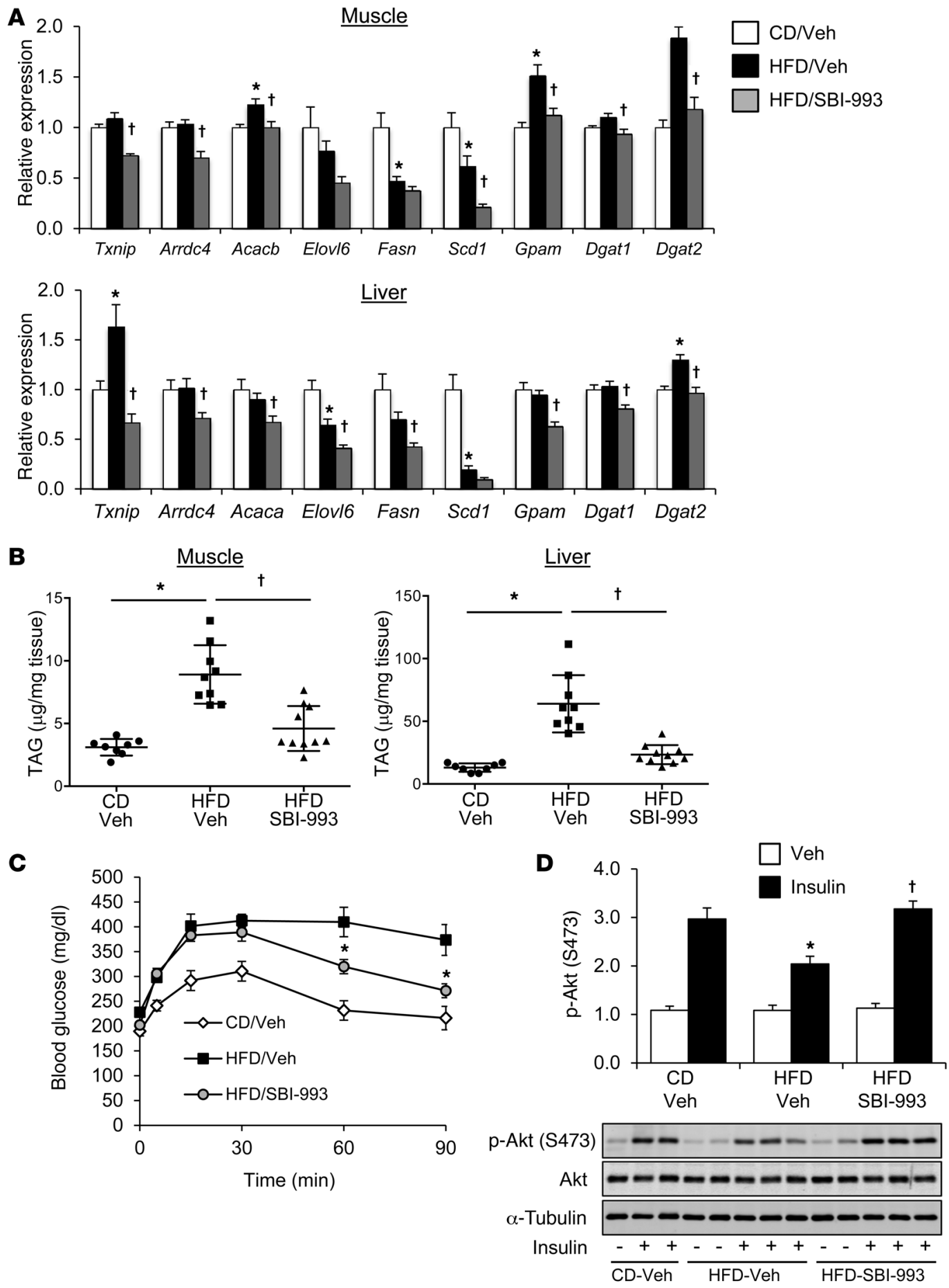

Figure 7. SBI-993 inhibits muscle and hepatic TAG accumulation and reduces MondoA target gene expression in vivo. Mice maintained on an HFD for 8 weeks were administered SBI-993 (50 mg/kg, q.d., s.c.) or a vehicle control for the final week of HFD feeding. Target gene expression (A) and total triglyceride content (B) were measured in skeletal muscle (gastrocnemius) and liver in mice maintained on a control diet (CD) or HFD ( $n=6-10$ mice per group). ${ }^{*} P<0.05$ versus $C D /$ vehicle; ${ }^{\dagger} P<0.05$ versus HFD/vehicle; 1 -way ANOVA with Bonferroni's post-hoc test. (C) Blood glucose levels are shown following a glucose tolerance test $(1 \mathrm{~g} / \mathrm{kg}$ glucose, i.p.) after dosing with SBI-993 or vehicle $(n=6$ per group). Data represent the mean \pm SEM. ${ }^{*} P<0.05 \mathrm{HFD} /$ vehicle versus HFD/SBI-993; 2-way ANOVA with Tukey's multiple comparisons post-hoc test. (D) Western blot analysis of gastrocnemius skeletal muscle whole-cell lysate from mice receiving an acute insulin challenge $(1.5 \mathrm{U} / \mathrm{kg}$ for 10 minutes) to examine insulin signaling using $\mathrm{p}$-Akt (S473). Graph shows quantification of Western blot analysis ( $n=4-6$ per condition). Representative Western blots are shown. ${ }^{*} P<0.05$ versus $\mathrm{CD} /$ vehicle; ${ }^{\dagger} P<0.05$ versus HFD/vehicle; 1 -way ANOVA with Bonferroni's post-hoc test. 


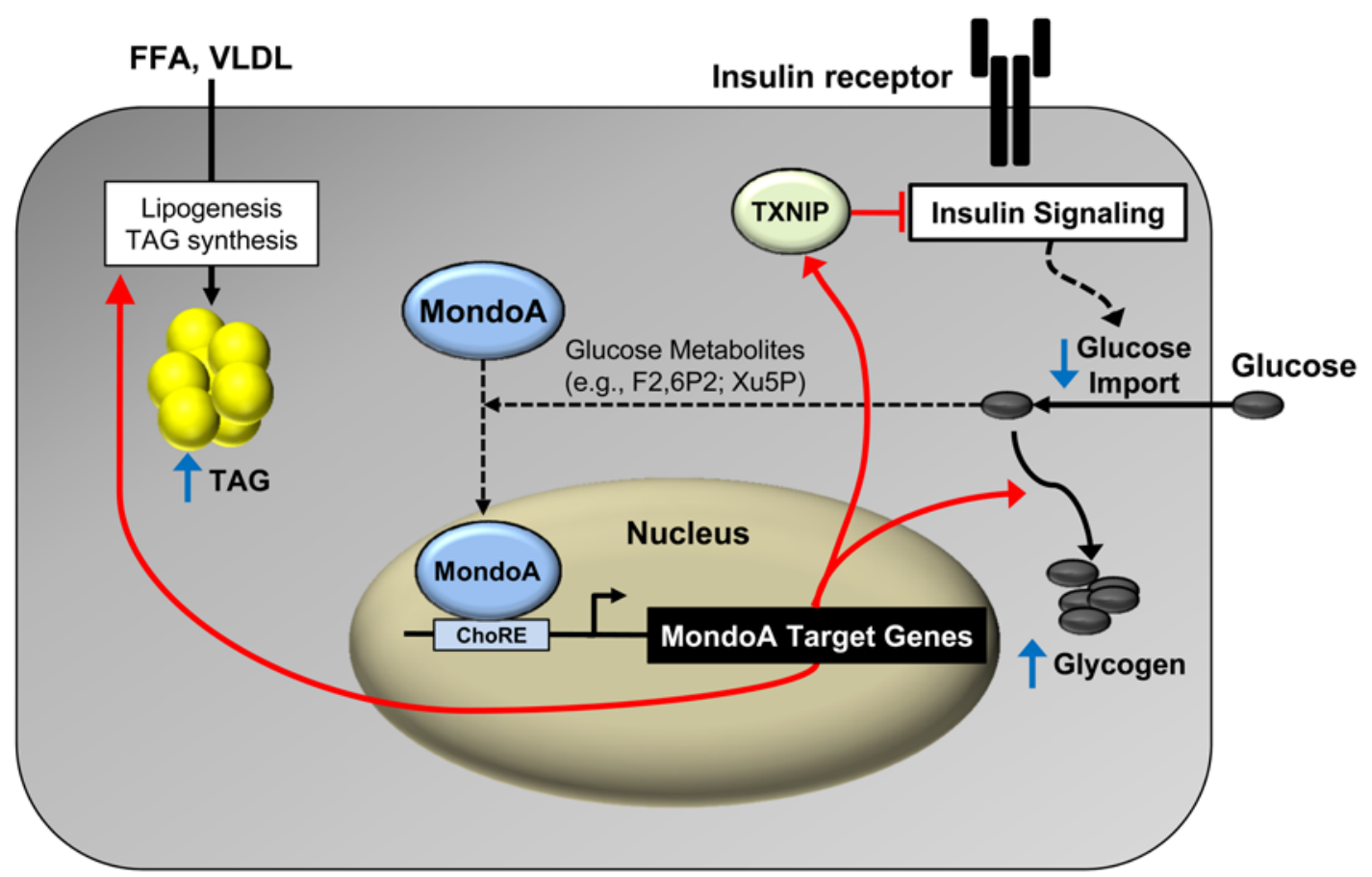

Figure 8. MondoA directs myocyte fuel homeostatic checkpoint functions. Proposed gene-regulatory (red arrows) and metabolic "checkpoint" responses (blue arrows) downstream of MondoA. MondoA is a glucose "sensor" that is directly activated by glycolytic metabolites that stimulate nuclear import of MondoA. Once activated, MondoA functions as a "brake" to limit carbon entry into the cell via increasing levels of TXNIP, an inhibitor of insulin signaling and glucose uptake. In addition, MondoA promotes energy storage through activation of enzymes involved in lipid and glycogen synthesis. Thus, MondoA may serve to limit carbon intake and fuel burning during conditions of "plenty." However, in states of chronic nutrient excess, persistent activation of MondoA may become maladaptive, contributing to a vicious cycle of cellular lipid accumulation (TAG synthesis) and insulin resistance (TXNIP-mediated suppressive effects).

lin challenge (Figure 7D and Supplemental Figure 8B). These data demonstrate that this compound class ameliorates obesity-related lipotoxicity, including lipid accumulation and glucose tolerance, concomitant with reduced MondoA/ChREBP signaling and improved insulin action.

\section{Discussion}

Excessive cellular neutral lipid accumulation is a hallmark of caloric excess and obesity. It is now well established that expansion of IMCL is strongly associated with the development of insulin resistance (2, 27-29). However, the intracellular lipid depot, per se, is probably not directly involved in this pathologic process, as insulin-sensitive conditions may also be associated with an IMCL increase such as that seen in endurance athletes (30). Although specific lipid species such as DAGs and ceramides have been shown to contribute to muscle insulin resistance in some studies (31-35), this conclusion has not been consistently supported by all the published literature (36-38). In addition, the normal biological processes that coordinately control myocyte lipid storage and glucose import are poorly understood. Therefore, we conducted an unbiased chemical biology screen to identify regulatory circuits that coordinately control myocyte lipid stores and glucose uptake. Delineation of the downstream actions of a molecule identified in this screen revealed that the transcription factor MondoA coordinately regulates genes involved in myocyte TAG synthesis and TXNIP and ARRDC4, which are known inhibitors of insulin signaling. Thus, inhibition of MondoA by SBI- 477 results in reduced cellular TAG accumulation and enhanced glucose uptake. This mechanism was shown to be operative in vivo in skeletal muscle and liver, suggesting that this regulatory pathway is relevant to multiple cell types.

MondoA was first described as a heterodimeric partner of the MLX transcription factor (24). It has been proposed that MondoA and its more intensively studied hepatic-enriched relative, ChREBP (MondoB), serve as intracellular glucose and energy sensors $(26,39,40)$. Increased levels of glucose-6-phosphate and other carbohydrate intermediates have been shown to stimulate the nuclear import of ChREBP and MondoA, resulting in a feedback loop that results in the control of glucose uptake and metabolism $(25,40-43)$. The results described herein demonstrate that MondoA activates the expression of genes involved in myocyte TAG synthesis and de novo lipogenesis. This regulation is reminiscent of the effects of ChREBP on hepatic lipogenesis $(44,45)$. Depletion of MondoA reduced TAG levels in oleate-loaded myocytes, an effect that mimicked the inhibitory effects of SBI-477. This mechanism was also shown in vivo, as administration of SBI-993 resulted in a reduction of muscle TAG levels and of hepatic steatosis, both of which are associated with reduced expression of lipogenic and TAG synthesis genes.

MondoA suppresses myocyte glucose uptake via activation of the $\alpha$-arrestin proteins TXNIP and ARRDC4, establishing a negative feedback loop to restrict glucose entry $(25,46)$. Overexpression of either TXNIP or ARRDC4 inhibits cellular glucose uptake $(20,47)$ by repressing insulin signaling via mechanisms 
that are as yet undetermined (21). Interestingly, TXNIP expression is increased in skeletal muscle of type 2 diabetes patients and inversely correlated with insulin-stimulated glucose uptake (20). In addition, deletion of Txnip in the $o b / o b$ background improved insulin sensitivity with activation of the insulin-signaling pathway in skeletal muscle (21). SBI-477 administration phenocopied the effects on TXNIP and ARRDC4 expression observed with MondoA depletion in skeletal myocytes, resulting in enhanced cellular glucose uptake. SBI-477 exerts this effect by markedly reducing nuclear levels of MondoA.

The MondoA-mediated mechanism described here was identified by a cell-based phenotypic small-molecule screen. Phenotypic screens allow for unbiased identification of molecules that function as probes of specific cellular processes and mechanisms with high relevance to biology and disease. However, a current challenge of phenotypic screens relates to the difficulty in identifying the direct molecular target. Accordingly, whereas this approach was powerful in that we identified what we believe to be a novel regulatory mechanism relevant to the control of myocyte lipid homeostasis and insulin resistance, the direct target remains unknown. It is possible that SBI-477 directly targets enzymes involved in de novo lipogenesis or TAG synthesis. We have ruled out obvious targets such as DGAT, MGAT, or SCD1, but cannot exclude the possibility that SBI-477 inhibits other enzymes in this or related pathways. However, our results are most consistent with a mechanism that imposes upstream control of MondoA signaling. It is tempting to speculate that SBI-477 affects the level of an intracellular signal that controls nuclear localization of both MondoA and ChREBP. Indeed, previous studies have identified several candidate mediators for the control of ChREBP/MondoA cellular localization and activity including intermediates of glucose metabolism, OGlcNAc modification, and phosphorylation $(40,42,48-50)$. However, given the impact of SBI-477 on lipid metabolism, it is also possible that a lipid signal influences MondoA nuclear localization.

The results described herein raise the question regarding what the normal biological role is of MondoA signaling in muscle. Given the published results and our findings, we propose that MondoA serves to maintain cellular carbon and energy homeostasis. Specifically, in states of acute fuel excess and ample energy stores, MondoA may serve to reduce fuel catabolism by triggering metabolic checkpoint functions that redirect carbon sources by inhibiting glucose uptake via suppression of insulin signaling and incorporation of FAs into lipid storage depots (Figure 8). MondoA has also been shown to activate genes involved in glycogen synthesis, further supporting a role for this protein in the diversion of fuel to storage depots (51). The known mechanisms of MondoA regulation are also consistent with this notion. For example, MondoA nuclear levels are increased by glucose metabolites, including phosphometabolites, which could serve as indicators of high glucose flux and ample energy phosphate stores $(51,52)$. In addition, inhibition of oxidative phosphorylation results in deactivation of MondoA, which would release its inhibition on glucose import and fuel catabolic flux (53). Accordingly, MondoA may serve to limit carbon intake and fuel burning during periods of acute fuel excess. We speculate, however, that in states of chronic caloric excess, persistent activation of MondoA becomes maladaptive (Figure 8), contributing to a vicious cycle of cellular lipid accumulation (TAG synthesis) and insulin resistance (TXNIP-mediated effects).

Our findings demonstrate that the regulatory circuit defined in the cell studies is operative in vivo. Following administration of an HFD to mice, a structural homolog of SBI-477, SBI-993, reduced TAG levels in muscle and, to a greater extent, in liver. The reduction of intramyocellular and hepatic TAG accumulation was associated with improved insulin signaling and glucose tolerance. It should be noted that the compound caused modest weight loss. We cannot exclude the possibility of some contribution to the metabolic effects of SBI-993 by the modest weightloss effects. Notably, the weight loss was not due to reduced food intake (data not shown), making a generalized toxic effect of the compound unlikely. In addition, the effects on tissue lipids and Txnip gene expression were disproportionate to the modest weight loss. Interestingly, the ChIP results demonstrated that SBI-993 reduced the occupation of ChREBP in addition to MondoA on liver target genes, suggesting that the compound probably affects both MondoA and ChREBP in liver. These latter results suggest that the relative contribution of MondoA and ChREBP downstream of SBI-477/993 depends on the cell and tissue context. Taken together, the findings provide a rationale for future studies aimed at validating this pathway as a therapeutic target for insulin resistance and tissue lipotoxicity caused by chronic caloric excess.

\section{Methods}

Cell culture. Primary human skeletal myoblasts, provided by Steven Smith (Translational Research Institute for Metabolism and Diabetes, Florida Hospital, Orlando, Florida, USA), were obtained by muscle biopsy from lean, healthy men and were isolated and cultured. Myoblasts were grown to a confluence of approximately $80 \%$ to $90 \%$ and differentiated into myotubes as previously described (54). Rat heart myoblast H9c2 cells (ATCC) were maintained in DMEM containing $10 \% \mathrm{FBS}$ in a $5 \% \mathrm{CO}_{2}$ incubator. At approximately $80 \%$ to $90 \%$ confluence, H9c2 myoblasts were differentiated into myotubes by culturing them in DMEM supplemented with $1 \%$ FBS for 4 to 5 days.

FAO assay. FAO rates were determined for primary human skeletal myotubes, with or without SBI-477, using $125 \mu \mathrm{M}\left[{ }^{3} \mathrm{H}\right]$-palmitic acid (55). Details can be found in the Supplemental Methods.

Microarray studies. Differentiated primary human myotubes were incubated with $10 \mu \mathrm{M}$ SBI-477, $1 \mu \mathrm{M}$ A922500 (DGAT inhibitor), or DMSO vehicle control for 24 hours. Total RNA was extracted using RNA-Bee (Amsbio) and cleaned up using the RNeasy Column (QIAGEN) following the manufacturer's protocol. The Genomics Core at SBP-LN performed the hybridization to a GeneChip Human gene 1.0 ST Array (Affymetrix). Two independent samples were analyzed for each group. Affymetrix Experiment Console, version 1.1, was used to normalize array data by the robust multichip averaging (RMA) approach. R package Linear Model for Microarray Data (LIMMA) was applied for differential gene expression analysis. The gene array data were deposited in the NCBI's Gene Expression Omnibus (GEO) database (GEO GSE77212).

Lipidomics. Quantitative lipidomic analyses were performed on human skeletal myotubes exposed to BSA or $100 \mu \mathrm{M}$ oleate in the 
presence of DMSO vehicle (white bars, Figure 2) or $10 \mu \mathrm{M} \mathrm{SBI}-477$ (black bars, Figure 2) for 24 hours. Details can be found in the Supplemental Methods.

Glycogen synthesis assay. Human skeletal myotubes were incubated with or without SBI-477 and then treated with or without insulin $(100 \mathrm{nM})$ for 30 minutes. Glycogen synthesis rates were then measured as described previously (56). Details can be found in the Supplemental Methods.

Glucose tolerance test and insulin signaling. Following a 5-hour fast, a blood sample was obtained from the tail tip for the measurement of baseline glucose levels using a hand-held glucometer (Accu-Chek Aviva Plus; Roche). A bolus of glucose ( $1 \mathrm{~g} / \mathrm{kg}$ ) was then administered to the mice via i.p. injection. Blood samples were obtained at 5, 15, 30, 60, and 90 minutes after the glucose bolus for measurement of blood glucose levels. For detection of insulin signaling, mice were fasted for 5 hours on the last day of the study. Gastrocnemius skeletal muscle and liver tissues were harvested 10 minutes after an acute i.p. insulin administration $(1.5 \mathrm{U} / \mathrm{kg})$ and immediately snap frozen. The tissue samples were homogenized in radioimmunoprecipitation assay (RIPA) buffer containing $1 \%$ NP-40, $5 \mathrm{mM} \mathrm{Na}_{4} \mathrm{P}_{2} \mathrm{O}_{7}, 1 \mathrm{mM}$ EDTA, $20 \mathrm{mM} \mathrm{NaF}, 2 \mathrm{mM} \mathrm{Na}_{3} \mathrm{VO}_{4}$, $1 \times$ cOmplete protease inhibitor (Roche), and $1 \mathrm{mM}$ PMSF. Western blot analysis to determine levels of p-Akt (S473) and total Akt was then performed.

SBI-477 and SBI-993 synthesis. Synthesis of SBI-477 and SBI-993 was performed as described in the Supplemental Methods.

Myocyte triglyceride measurements. Human skeletal myotubes were differentiated for 8 days. On day 7, oleic acid $(100 \mu \mathrm{M})$ complexed to FA-free BSA was added to the cells with the test compound for 24 hours. Following incubation with oleate and compound, cells were formaldehyde fixed and stained with AdipoRed (Lonza). TAG accumulation was measured using signal intensity at excitation 540/ emission $590 \mathrm{~nm}$ (BioTek Instruments). Dose-response curves were generated in GraphPad Prism 6 (GraphPad Software). EC $_{50}$ values were calculated using nonlinear regression analysis. For biochemical measurement of TAGs, human skeletal myotubes were incubated with oleate-BSA and test compound as described above. After a 24-hour incubation, cells were collected with a lysis buffer containing 0.1\% IGEPAL CA-630 (Sigma-Aldrich) in PBS. The harvested cells were sonicated for 5 seconds and then centrifuged at 10,000 $g$ for 10 minutes at $4^{\circ} \mathrm{C}$. The supernatant was removed and stored on ice. TAG levels were quantified using Infinity Triglycerides Liquid Stable Reagent (Thermo Fisher Scientific) according to the manufacturer's instructions for colorimetric assay.

Immunoblot analysis. Cells were lysed in the RIPA buffer containing 1\% NP-40, $5 \mathrm{mM} \mathrm{Na}_{4} \mathrm{P}_{2} \mathrm{O}_{7}, 1 \mathrm{mM}$ EDTA, $20 \mathrm{mM} \mathrm{NaF}, 2 \mathrm{mM}$ $\mathrm{Na}_{3} \mathrm{VO}_{4}, 1 \times$ cOmplete Protease Inhibitor (Roche), and $1 \mathrm{mM}$ PMSF. Whole-cell lysates were subjected to SDS-PAGE and transferred to a nitrocellulose membrane. The blots were hybridized with specific Abs and developed with an ECL kit (Pierce, Thermo Fisher Scientific). The following Abs were used: p-Akt (S473); Akt; p-IRS-1 (S636/639); S6K; p-S6K (T389); AMPK $\alpha$; p-AMPK $\alpha$ (T172); $\alpha$-tubulin; lamin A/C (Cell Signaling Technology; catalogs 4058, 9272, 2388,9202 , 9234, 2532, 2531, 3873, and 2032, respectively); $\beta$-actin and IRS-1 (Y612) (Sigma-Aldrich; catalogs A5316 and I2658); IRS-1 and GAPDH (Santa Cruz Biotechnology Inc.; catalogs SC-560 and SC-25778); TXNIP (MBL; catalog K0205-3); and MondoA (Bethyl
Laboratories; catalog A303-195A). Protein quantification was performed using FluorChemQ (Alpha Innotech) and normalized to $\beta$-actin or total protein.

Cellular glucose uptake assay. After differentiation, primary human skeletal myotubes were incubated with SBI- 477 for 24 hours and then treated with insulin $(100 \mathrm{nM})$ for 30 minutes. Cells were washed in PBS 3 times at room temperature and then incubated with Krebs-Ringer HEPES buffer (140 mM NaCl, $5 \mathrm{mM} \mathrm{KCl,} 1 \mathrm{mM} \mathrm{CaCl}_{2}, 2.5 \mathrm{mM}$ $\mathrm{MgSO}_{4}, 2.5 \mathrm{mM}, \mathrm{NaH}_{2} \mathrm{PO}_{4}, 20 \mathrm{mM}$ HEPES, and 0.1\% BSA) containing $\left[{ }^{3} \mathrm{H}\right]$-2-deoxyglucose (2-DG) $(1.0 \mu \mathrm{Ci} / \mathrm{ml})$ for 15 minutes. Glucose uptake was terminated by washing 5 times with ice-cold PBS. The cells were then solubilized with $0.5 \mathrm{~N}$ sodium hydroxide $(\mathrm{NaOH})$. The amount of 2-DG taken up was measured by liquid scintillation analyzer (PerkinElmer) and normalized to total protein.

RNA isolation and quantitative RT-PCR. Total RNA was isolated using an RNeasy Mini Kit (QIAGEN) according to the manufacturer's instructions. cDNA was synthesized using the Affinity Script cDNA Synthesis Kit (Stratagene) with $0.5 \mu$ g total RNA. PCR reactions were performed in triplicate (Roche LightCycler 480 Instrument II) with specific primers for each gene. Primer sets are listed in Supplemental Table 4. The expression of RplpO (also known as 36b4) was used to normalize all gene expression data.

Transient transfection and luciferase reporter assay. Transient transfections were performed using Attractene (QIAGEN) according to the manufacturer's protocol. A full-length (1.5-kb) and 2 serial deletion (1.0$\mathrm{kb}$ and $0.5-\mathrm{kb}$ ) reporters of the human TXNIP promoter were provided by Fumihiko Urano (Washington University School of Medicine, St. Louis, Missouri, USA). Mutation of the ChoREs of the reporters was generated by a QuikChange II Site-Directed Mutagenesis Kit (Agilent Technologies). H9c2 myoblast cells (ATCC) were cotransfected with $700 \mathrm{ng}$ of the reporter and $25 \mathrm{ng}$ of the CMV promoter-driven Renilla luciferase as a control for transfection efficiency. Eighteen hours after transfection, the cells were cultured in DMEM supplemented with 1\% FBS to induce differentiation for 4 days. SBI-477 was added during the last 24 hours. After compound incubation, luciferase activity was measured using the Dual-Glo Luciferase Assay System (Promega) according to the manufacturer's protocols.

ChIP assays. ChIP assays were performed as previously described (57). Briefly, differentiated human skeletal myotubes were cross-linked with $1 \%$ formaldehyde for 10 minutes at room temperature, and glycine was added to stop the cross-linking reaction. Cells were harvested and lysed. For chromatin fragmentation, sonication was performed using a Bioruptor (Diagenode). Proteins were immunoprecipitated using anti-MondoA (Bethyl Laboratories; catalog A303-195A) or IgG control (Sigma-Aldrich; catalog I5006) overnight at $4^{\circ} \mathrm{C}$. DNA fragments were purified using a QIAquick PCR Purification Kit (QIAGEN) and quantified with a LightCycler 480 Instrument II (Roche) with the specific primers listed in Supplemental Table 5.

In vivo studies. Six-week-old male C57BL/6J mice were obtained from The Jackson Laboratory (stock 000664) and acclimated for 1 week before the study. Mice were maintained on standard chow or a 60\% HFD (Research Diets) for 8 weeks. SBI-993 was dissolved in a vehicle containing 2\% DMSO, 2\% Tween-80, and 96\% water (pH 9.0). Seven weeks after HFD feeding, mice were s.c. injected with SBI993 (50 mg/kg mouse BW) once daily for 7 days. Each group had 6-10 mice, and 3 independent experiments were conducted. 
Statistics. The Student's $t$ test, Mann-Whitney $U$ test, 1-way ANOVA with Bonferroni's post-hoc test, or 2-way ANOVA with Tukey's post-hoc test was performed to determine statistical significance as indicated in the figure legends. Nonlinear regression analysis was used to calculate EC $_{50}$ values of SBI-477 and SBI-993 in the TAG accumulation assay in human skeletal myotubes (GraphPad Prism 6; GraphPad Software).

Study approval. All animal studies were performed in accordance with NIH guidelines for the humane treatment of animals and approved by the IACUC of the Sanford Burnham Prebys Medical Discovery Institute IACUC.

\section{Author contributions}

Conceptualization: BA, RBV, and DPK. Methodology: BA and MW. Investigation: BA, MMS, HS, SP, ES, AK, MW, KY, JB, and JLL. Data curation: BA, HS, SM, TCL, and RBV. Formal analysis: BA, HS, XH, and RBV. Writing of original draft: BA, RBV, and DPK. Writing - review and editing: BA, HS, XH, TCL, GPR, RBV, and DPK. Funding acquisition: GPR, XH, and DPK. Supervision: SM, GPR, XH, RBV, and DPK.

\section{Acknowledgments}

We dedicate this manuscript to the memory of Gregory Roth. We thank Donald Ayer, Timothy Osborne, and Peter Phelan for helpful discussions; Lorenzo Thomas for assistance with manuscript preparation; David Terry for measurements of SBI-993 compound exposure; Julio Ayala and the Cardiometabolic Phenotyping Core for assistance with the glucose tolerance tests; Feng Qi, Subramaniam Shyamalagovindarajan, and the Bioinformatics and Genomics Cores at SBP-LN for assistance with microarray studies; Ling Lai, Orlando Rodriguez, and Caron Stonebrook for assistance with the animal studies; and William Esler and Jeff Pfefferkorn (Pfizer Inc.) for assistance with discussion and measurements of DGAT1/2 and MGAT1/2/3 activity. This work was supported by NIH grants R01 DK045416, R24 DK092781, and R24 DK084969 (to DPK).

Address correspondence to: Daniel P. Kelly, Sanford Burnham Prebys Medical Discovery Institute at Lake Nona, 6400 Sanger Road, Orlando, Florida 32827, USA. Phone: 407.745.2096; E-mail: dkelly@sbpdiscovery.org.
1. Pan DA, et al. Skeletal muscle triglyceride levels are inversely related to insulin action. Diabetes. 1997;46(6):983-988.

2. Krssak M, et al. Intramyocellular lipid concentrations are correlated with insulin sensitivity in humans: a 1H NMR spectroscopy study. Diabetologia. 1999;42(1):113-116.

3. Goodpaster BH, Theriault R, Watkins SC, Kelley DE. Intramuscular lipid content is increased in obesity and decreased by weight loss. Metab Clin Exp. 2000;49(4):467-472.

4. Mitra R, et al. The transcriptional coactivators, PGC- $1 \alpha$ and $\beta$, cooperate to maintain cardiac mitochondrial function during the early stages of insulin resistance. JMol Cell Cardiol. 2012;52(3):701-710.

5. Petersen KF, Dufour S, Befroy D, Garcia R, Shulman GI. Impaired mitochondrial activity in the insulin-resistant offspring of patients with type 2 diabetes. NEngl J Med. 2004;350(7):664-671.

6. Schrauwen-Hinderling VB, et al. Impaired in vivo mitochondrial function but similar intramyocellular lipid content in patients with type 2 diabetes mellitus and BMI-matched control subjects. Diabetologia. 2007;50(1):113-120.

7. Phielix E, et al. Lower intrinsic ADP-stimulated mitochondrial respiration underlies in vivo mitochondrial dysfunction in muscle of male type 2 diabetic patients. Diabetes. 2008;57(11):2943-2949.

8. Listenberger LL, et al. Triglyceride accumulation protects against fatty acid-induced lipotoxicity. Proc Natl Acad Sci U S A. 2003;100(6):3077-3082.

9. Liu L, et al. DGAT1 expression increases heart triglyceride content but ameliorates lipotoxicity. J Biol Chem. 2009;284(52):36312-36323.

10. Samuel VT, et al. Mechanism of hepatic insulin resistance in non-alcoholic fatty liver disease. J Biol Chem. 2004;279(31):32345-32353.

11. Bosma M, Kersten S, Hesselink MK, Schrauwen P. Re-evaluating lipotoxic triggers in skeletal muscle: relating intramyocellular lipid metabolism to insulin sensitivity. Prog Lipid Res.
2012;51(1):36-49.

12. Idris I, Gray S, Donnelly R. Insulin action in skeletal muscle: isozyme-specific effects of protein kinase C. Ann N Y Acad Sci. 2002;967:176-182.

13. Kumashiro N, et al. Cellular mechanism of insulin resistance in nonalcoholic fatty liver disease. Proc Natl Acad Sci U S A. 2011;108(39):16381-16385.

14. Chavez JA, et al. A role for ceramide, but not diacylglycerol, in the antagonism of insulin signal transduction by saturated fatty acids. J Biol Chem . 2003;278(12):10297-10303.

15. Anderson EJ, et al. Mitochondrial $\mathrm{H} 2 \mathrm{O} 2$ emission and cellular redox state link excess fat intake to insulin resistance in both rodents and humans. JClin Invest. 2009;119(3):573-581.

16. Koves TR, et al. Mitochondrial overload and incomplete fatty acid oxidation contribute to skeletal muscle insulin resistance. Cell Metab. 2008;7(1):45-56.

17. Zou J, et al. Potent inhibitors of lipid droplet formation. In: Probe Reports from the NIH Molecular Libraries Program [Internet]. Bethesda, MD; National Center for Biotechnology Information (US); 2010-2011.

18. Han RH, Wang M, Fang X, Han X. Simulation of triacylglycerol ion profiles: bioinformatics for interpretation of triacylglycerol biosynthesis. JLipid Res. 2013;54(4):1023-1032.

19. Bouzakri K, et al. Reduced activation of phosphatidylinositol-3 kinase and increased serine 636 phosphorylation of insulin receptor substrate-1 in primary culture of skeletal muscle cells from patients with type 2 diabetes. Diabetes. 2003;52(6):1319-1325.

20. Parikh H, et al. TXNIP regulates peripheral glucose metabolism in humans. PLoS Med. 2007;4(5):e158

21. Yoshihara E, et al. Disruption of TBP-2 ameliorates insulin sensitivity and secretion without affecting obesity. Nat Commun. 2010;1:127.

22. Oslowski CM, et al. Thioredoxin-interacting protein mediates ER stress-induced $\beta$ cell death through initiation of the inflammasome. Cell Metab. 2012;16(2):265-273.

23. Cha-Molstad H, Saxena G, Chen J, Shalev A. Glucose-stimulated expression of Txnip is mediated by carbohydrate response element-binding protein, p300, and histone $\mathrm{H} 4$ acetylation in pancreatic beta cells. J Biol Chem 2009;284(25):16898-16905.

24. Billin AN, Eilers AL, Coulter KL, Logan JS, Ayer DE. MondoA, a novel basic helix-loop-helix-leucine zipper transcriptional activator that constitutes a positive branch of a max-like network. Mol Cell Biol. 2000;20(23):8845-8854.

25. Stoltzman CA, Peterson CW, Breen KT, Muoio DM, Billin AN, Ayer DE. Glucose sensing by MondoA:Mlx complexes: a role for hexokinases and direct regulation of thioredoxin-interacting protein expression. Proc Natl Acad Sci US A. 2008;105(19):6912-6917.

26. Filhoulaud G, Guilmeau S, Dentin R, Girard J, Postic C. Novel insights into ChREBP regulation and function. Trends Endocrinol Metab. 2013;24(5):257-268.

27. Pan DA, et al. Skeletal muscle membrane lipid composition is related to adiposity and insulin action. J Clin Invest. 1995;96(6):2802-2808.

28. Jacob S, et al. Association of increased intramyocellular lipid content with insulin resistance in lean nondiabetic offspring of type 2 diabetic subjects. Diabetes. 1999;48(5):1113-1119.

29. Coen PM, Goodpaster BH. Role of intramyocelluar lipids in human health. Trends Endocrinol Metab. 2012;23(8):391-398.

30. Goodpaster BH, He J, Watkins S, Kelley DE. Skeletal muscle lipid content and insulin resistance: evidence for a paradox in endurance-trained athletes. JClin Endocrinol Metab. 2001;86(12):5755-5761.

31. Montell E, et al. DAG accumulation from saturated fatty acids desensitizes insulin stimulation of glucose uptake in muscle cells. Am J Physiol Endocrinol Metab. 2001;280(2):E229-E237. 
32. Yu C, et al. Mechanism by which fatty acids inhibit insulin activation of insulin receptor substrate-1 (IRS-1)-associated phosphatidylinositol 3-kinase activity in muscle. J Biol Chem. 2002;277(52):50230-50236.

33. Adams JM, et al. Ceramide content is increased in skeletal muscle from obese insulin-resistant humans. Diabetes. 2004;53(1):25-31.

34. Bergman BC, Hunerdosse DM, Kerege A, Playdon MC, Perreault L. Localisation and composition of skeletal muscle diacylglycerol predicts insulin resistance in humans. Diabetologia. 2012;55(4):1140-1150.

35. Szendroedi J, et al. Role of diacylglycerol activation of PKC $\theta$ in lipid-induced muscle insulin resistance in humans. Proc Natl Acad Sci U S A. 2014;111(26):9597-9602.

36. Skovbro M, et al. Human skeletal muscle ceramide content is not a major factor in muscle insulin sensitivity. Diabetologia. 2008;51(7):1253-1260.

37. Anastasiou CA, Kavouras SA, Lentzas Y, Gova A, Sidossis LS, Melidonis A. Diabetes mellitus is associated with increased intramyocellular triglyceride, but not diglyceride, content in obese humans. Metab Clin Exp. 2009;58(11):1636-1642.

38. Amati F, et al. Skeletal muscle triglycerides, diacylglycerols, and ceramides in insulin resistance: another paradox in endurance-trained athletes?. Diabetes. 2011;60(10):2588-2597.

39. Dentin R, et al. Hepatic glucokinase is required for the synergistic action of ChREBP and SREBP$1 c$ on glycolytic and lipogenic gene expression. J Biol Chem. 2004;279(19):20314-20326.

40. Peterson CW, Stoltzman CA, Sighinolfi MP, Han KS, Ayer DE. Glucose controls nuclear accumulation, promoter binding, and transcriptional activity of the MondoA-Mlx heterodimer. Mol Cell Biol. 2010;30(12):2887-2895.
41. Kabashima T, Kawaguchi T, Wadzinski BE, Uyeda K. Xylulose 5-phosphate mediates glucoseinduced lipogenesis by xylulose 5 -phosphateactivated protein phosphatase in rat liver. Proc Natl Acad Sci U S A. 2003;100(9):5107-5112.

42. Sakiyama H, et al. Regulation of nuclear import/ export of carbohydrate response element-binding protein (ChREBP): interaction of an alpha-helix of ChREBP with the 14-3-3 proteins and regulation by phosphorylation. J Biol Chem. 2008;283(36):24899-24908.

43. Stoltzman CA, Kaadige MR, Peterson CW, Ayer DE. MondoA senses non-glucose sugars: regulation of thioredoxin-interacting protein (TXNIP) and the hexose transport curb. JBiol Chem. 2011;286(44):38027-38034.

44. Dentin R, et al. Liver-specific inhibition of ChREBP improves hepatic steatosis and insulin resistance in ob/ob mice. Diabetes. 2006;55(8):2159-2170.

45. Iizuka K, Miller B, Uyeda K. Deficiency of carbohydrate-activated transcription factor ChREBP prevents obesity and improves plasma glucose control in leptin-deficient (ob/ob) mice. Am J Physiol Endocrinol Metab. 2006;291(2):E358-E364.

46. Kaadige MR, Looper RE, Kamalanaadhan S, Ayer DE. Glutamine-dependent anapleurosis dictates glucose uptake and cell growth by regulating MondoA transcriptional activity. Proc Natl Acad Sci US A. 2009;106(35):14878-14883.

47. Patwari P, et al. Thioredoxin-independent regulation of metabolism by the alpha-arrestin proteins. J Biol Chem. 2009;284(37):24996-25003.

48. Kawaguchi T, Takenoshita M, Kabashima T, Uyeda K. Glucose and cAMP regulate the L-type pyruvate kinase gene by phosphorylation/ dephosphorylation of the carbohydrate response element binding protein. Proc Natl Acad Sci U S A. 2001;98(24):13710-13715.

49. Bricambert J, Miranda J, Benhamed F, Girard J, Postic C, Dentin R. Salt-inducible kinase 2 links transcriptional coactivator $\mathrm{p} 300$ phosphorylation to the prevention of ChREBPdependent hepatic steatosis in mice. JClin Invest. 2010;120(12):4316-4331.

50. Guinez C, et al. O-GlcNAcylation increases ChREBP protein content and transcriptional activity in the liver. Diabetes. 2011; 60(5):1399-1413.

51. Petrie JL, et al. Glucose induces protein targeting to glycogen in hepatocytes by fructose 2,6-bisphosphate-mediated recruitment of MondoA to the promoter. Mol Cell Biol. 2013;33(4):725-738.

52. Sloan EJ, Ayer DE. Myc, mondo, and metabolism. Genes Cancer. 2010;1(6):587-596.

53. Yu FX, Chai TF, He H, Hagen T, Luo Y. Thioredoxin-interacting protein (Txnip) gene expression: sensing oxidative phosphorylation status and glycolytic rate. J Biol Chem. 2010;285(33):25822-25830.

54. Sparks LM, et al. Remodeling lipid metabolism and improving insulin responsiveness in human primary myotubes. PLOS ONE. 2011;6(7):e21068.

55. Djouadi F, Bonnefont JP, Munnich A, Bastin J. Characterization of fatty acid oxidation in human muscle mitochondria and myoblasts. Mol Genet Metab. 2003;78(2):112-118.

56. Halse R, Rochford JJ, McCormack JG, Vandenheede JR, Hemmings BA, Yeaman SJ. Control of glycogen synthesis in cultured human muscle cells. J Biol Chem. 1999;274(2):776-780.

57. Gan Z, et al. The nuclear receptor PPAR $\beta / \delta$ programs muscle glucose metabolism in cooperation with AMPK and MEF2. Genes Dev. 2011;25(24):2619-2630. 